\title{
N?2 30129
}

\section{SPACE RESEARCH COORDINATION CENTER}

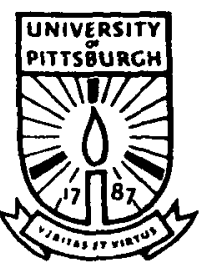

\section{MOBILITIES OF URANIUM AND MERCURY IONS IN HELIUM}

\author{
SPONSORED BY \\ ADV ANCED RESEARCH PROJECTS AGENCY \\ ARPA ORDER NO. 826 \\ BY
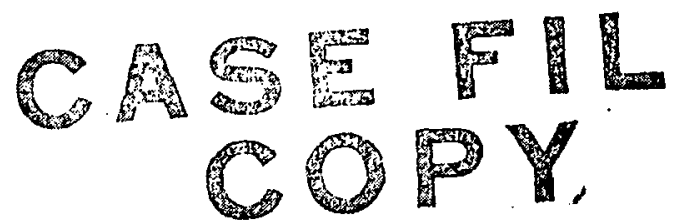

R. JOHNSEN AND MANFRED A. BIONDI

APPROVED FOR PUBLIC RELEASE;

DISTRIBUTION UNLIMITED

SRCC REPORT NO. 177

AUGUST 1972

UNIVERSITY OF PITTSBURGH

PITTSBURGH, PENNSYLVANIA

THE VIEWS AND CONCLUSIONS CONTAINED IN THIS DOCUMENT ARE THOSE OF THE AUTHORS AND SHOULO NOT BE INTERPRETED AS NECESSARILY REPRESENTING THE OFFICIAL POLICIES, EITHER EXPRESSED OR IMPLIED, OF THE ADVANCED RESEARCH PROJECTS AGENCY OR THE U.S. GOVERNMENT. 
The Space Research Coordination Center, established in May, 1963, has the following functions: (1) it administers predoctoral and postdoctoral fellowships in space-related science and engineering programs; (2) it makes avallable, on a pplication and after review, allocations to assist new faculty members in the Division of the N a tural Sciences and the School of Engineering to Initiate research programs or to permit established faculty members to do preliminary; work on research Ideas of a novel character; (3) in the Division of the Natural Sciences it makes an annual allocation of funds to the interdisciplinary Laboratory for Atmospheric and Space Sciences; (4) in the School of Engineering it makes a similar allocation of funds to the Department of Metallurgical and Materials Engineering and to the program in Engineering Systems Management of the Department of Industrial Engineering; and (5) in concert with the University's Knowledge Avallabllity Systems Center, it seeks to aseist In the orderly transfer of new space-generated knowledge in industrial application. The Center also issues periodic reports of space-oriented research and a comprehensive annual report.

The Center is supported by an Institutional Grant (NsG-416) from the National Aeronautics and Space Administration. strongly supplemented by grants from the A.W. Mellon Educational and Charitable Trust, the Maurice Falk Medical Fund, the Pachard King Mellon Foundation and the Sarah Mellon Scaife Foundation. Much of the work described in SRCC reports is financed by other grants, made to individual faculty members. 


\title{
Mobilities of Uranium and Mercury Ions in Helium
}

R. Johnsen and Manfred A. Biondi

(Journal of Chemical Physics)

\author{
Sponsored by \\ Advanced Research Projects Agency \\ ARPA Order No. 826
}

Approved for public release;

distribution unlimited

University of Pittsburgh

Pittsburgh, Pennsylvania

August 1972

The views and conclusions contained in this document are those of the authors and should not be interpreted as necessarily representing the official policies, either expressed or implied, of the Advanced Research Projects Agency or the U.S. Government. 
Mobilities of Uranium and Mercury Ions in Helium*

\author{
R. Johnsen and Manfred A. Biondi \\ Department of Physics, University of Pittsburgh \\ Pittsburgh, Pennsylvania 15213
}

\begin{abstract}
The mobilities of mass-identified $\mathrm{U}^{+}$and $\mathrm{Hg}^{+}$ions in helium have been determined in a drift tube-mass spectrometer apparatus. For uranium Ions a reduced mobility value $\mu_{0}=(16.0 \pm 0.5) \mathrm{cm}^{2} / \mathrm{V}-\mathrm{sec}$ is obtained at $305^{\circ} \mathrm{K}$ and a standard gas density of $2.69 \times 10^{19} \mathrm{~cm}^{-3}$. The mobility of mercury ions is $\mu_{0}=(19.4 \pm 0.5) \mathrm{cm}^{2} / \mathrm{V}-\mathrm{sec}$ at $292^{\circ} \mathrm{K}$, in agreement with two previous determinations. The effect of fast ion injection in drift mobility measurements is discussed, and a new technique to circumvent these problems is described. The results are compared with existing theories of ion mobilities.
\end{abstract}

*

This research was supported by the Advanced Research Projects Agency of the Department of Defense and was monitored by U.S. Army Research OfficeDurham under Contract No. DA-31-124-ARO-D-440. 


\section{Introduction}

Ionic mobilities in rare gas have been of both experimental and theoretical interest for a long while, and a rather large body of data is available for comparison with theory. We have recently carried out some drift tube measurements of ion-molecule reactions of uranium ions with atmospheric gases ${ }^{(1)}$, for which accurate determinations of the mobility of uranium ions in helium (used as a buffer gas in that experiment) are necessary. The results of these ion mobility determinations and some of the special problems encountered in mobility measurements of heavy ions in light gases are the subjects of the present article. In addition, we present our measurements of the mobility of mercury ions in helium (for which two independent earlier determinations exist $(2,3)$ ) which were carried out to test the validity of the experimental procedures used.

\section{Method of Measurement}

The drift tube-mass spectrometer apparatus and the electron impact ion source for production of uranium ions shown in Fig. I have been described earlier(1). A pulse of ions lasting typically 5-10 $\mu \mathrm{sec}$ is injected from the source into the uniform field region of the drift tube. A fraction of the ions which remain on axis in traversing the drift region (length, $15.75 \mathrm{~cm}$ ) effuse through the small exit orifice into the differentially pumped quadrupole mass spectrometer, where they are mass-identified and counted with the aid of a channeltron electron multiplier. The counts at each arrival time interval are stored in a multichannel analyzer operating in a time-of-flight mode.

In the present experiment, a water cooled jacket surrounding the ion source and the flange connecting it to the drift tube has been added 
to eliminate heating of the drift tube by the evaporator filament which dissipates about $150 \mathrm{~W}$ of electrical power. With these precautions the temperature of the drift tube vacuum enclosure remained at about $29^{\circ} \mathrm{C}$, and no effect on the gas density was detected when the evaporator was turned on. Furthermore, no effect of getter pumping of helium by the uranium evaporator was noted. (By contrast, attempts to measure the mobility of uranium ions in nitrogen were abandoned because the nitrogen pressure in the drift region dropped from about 0.2 Torr to one hundredth of that value only seconds after the evaporator was turned on.)

For the mercury ion studies, the evaporator filament was coated with mercury sulfate powder, which decomposes to provide mercury vapor upon heating. In order to prevent contamination of the gas handling vacuum system by mercury vapor, a dry ice cold trap was installed in the gas inlet lines to the ion source.

The mobility is determined from measurements of the transit time of a pulse of ions through the drift tube at a known applied electric field and gas pressure. As a result of diffusion the arrival times of individual ions are distributed about the average transit time. The time of maximum ion signal for this type of drift tube usually is within $1 / 2 \%$ of the average transit time and was therefore used without correction in the analysis of the data. The time that the ions spend traversing the mass spectrometer was both calculated and measured to be $40 \mu \mathrm{sec}$ and has been subtracted from the measured total transit times.

The ions are extracted from the ion source by means of an electric field substantially larger than that present in the drift tube. As a result of the inefficiency of momentum exchange between heavy ions and light helium atoms, the ions may penetrate deeply into the drift tube before reaching the stationary energy distribution appropriate to the applied drift field and the 
gas temperature. This "injection effect" leads to a reduction of the effective length of the drift region by an unknown amount and thus causes the measured mobilities to be too high. An estimate for the "depth of injection" of ions injected with an initial velocity $v_{0}$ into a gas of pressure $p$ may be obtained by multiplying the initial velocity by the time constant $\tau$ for the relaxation of the drift velocity derived in a previous paper( ${ }^{(1)}$. The resulting depth of injection 8 then becomes

$$
\delta(\mathrm{cm})=1.2 \times 10^{-3}\left(\mu_{0} / \mathrm{p}\right)\left(M \varepsilon_{0}\right)^{\frac{2}{2}},
$$

where $\mu_{0}$ is the reduced mobility in $\mathrm{cm}^{2} / \mathrm{V}-\mathrm{sec}, \mathrm{p}$ the pressure in Torr, $M$ the mass of the ion in atomic units, and $\varepsilon_{0}$ the initial energy.

Equation (I) appears to give qualitatively correct values of the injection depth, since at an injection energy of $\sim 100 \mathrm{eV}$ and a. He gas pressure of 0.35 Torr an apparent $U^{+}$ion mobilitivy of almost twice the correct value is measured. This indicates an injection depth of about half the $15.75 \mathrm{~cm}$ drift length, in agreement with the value $(\sim 8 \mathrm{~cm})$ calculated from the equation. While the example chosen is an extreme case, it does illustrate the appreciable penetration of heavy ions injected from a source into a light gas. Thus, accurate mobility measurements should be carried out using techniques that do not depend on a fixed drift region length, for example differential drift distance techniques $(4,5)$.

Since our apparatus does not permit us to vary the length of the drift region, an alternate solution has been devised. Subsequent to the injection of lons into the drift tube, the electric field in the tube is reversed, with the result that the ions return to the entrance plate, where most of them are neutralized by striking the metal. After the normal field direction is restored, 
the few remaining ions traverse the drift tube with an energy determined by the drift field; their effective point of origin is the entrance plate. Proper operation of this technique has been checked by increasing the magnitude and duration of the reversed field pulse. The height and length of this pulse are considered adequate when a further increase in either quantity reduced the ion signal but does not effect a change in the measured ion transit time.

In addition to the reversed field technique, measurements were carried out in conventional fashion but at very low ion injection energies, approaching the limit where the ions diffuse into the drift tube at thermal energy. This technique, although suffering from very poor signal strength and brozder ion arrival waveforms; gave results which agreed with the results obtained by the reversal method to within 5\% (for gas pressures 21 Torr)。

\section{Results}

Following convention we express our results in terms of the reduced mobility $\mu_{0}$ at a standard gas density of $2.69 \times 10^{19} \mathrm{~cm}^{-3}$. The mobilities are measured as a function of $\mathrm{E} / \mathrm{p}$, where $\mathrm{E}$ is the drift field, and at a gas temperature of $\sim 300^{\circ} \mathrm{K}$.

The reduced mobilities of $\mathrm{U}^{+}$ions in helium are shown by the lower curve in Fig. 2. In these measurements the reversed drift field technique was used to eliminate errors due to ion injection effects. Extrapolation of the data to $\mathrm{E} / \mathrm{p}=0$ yields a thermal energy value of $\mu_{0}=(16.0 \pm 0.5) \mathrm{cm}^{2} / \mathrm{V}$-sec.

The $\mathrm{Hg}^{+}$mobility determinations exhibited less pronounced ion injection effects than was the case for the uranium studies. It appears that most of the mercury ions are formed indirectly by Penning ionization from metastable helium atoms and by charge transfer from helium ions rather than by electron impact ionization in the source. These processes probably take place in the immediate vicinity of the injection orifice (and in the drift region)。 so that the mercury ions do not traverse the accelerating potential in the ion source. 
To duplicate the uranium measurements as closely as possible, the drift field was reversed for a short time after injection of an ion pulse. This procedure has the additional advantage that the helium ions and metastable atoms have sufficient time to decay before the normal drift field direction is restored. Thus, production of mercury ions in the drift region (which could affect the mobility measurements) is minimized.

The measured values of the reduced mobility of $\mathrm{Hg}^{+}$ions in helium are shown by the upper curve in Fig. 2. The gas temperature in these measurements is $292^{\circ} \mathrm{K}$. The extrapolated zero field mobility is $\mu_{0}=$ $(19.4 \pm 0.5) \mathrm{cm}^{2} / \mathrm{V}-\mathrm{sec}$.

IV. Discussion and Conclusions

The measured values of the reduced mobilities of uranium and mercury ions in helium may be compared with other measurements and with existing theories. The measured thermal energy value for $\mathrm{Hg}^{+}$ions in helium, Ho $=$ $(19.4 \pm 0.5) \mathrm{cm}^{2} / \mathrm{V}-\mathrm{sec}$ is in excellent agreement with the results of Biondi's microwave afterglow determination(2) of the ambipolar diffusion coefficient of mercury ions in helium which yielded the value $\mu_{0}=(19.8 \pm 1) \mathrm{cm}^{2} / \mathrm{V}-\mathrm{sec}$. It is also in excellent agreement with Chanin and Biondi's mobility tube determination(3), employing differential drift distance techniques, which yielded the value $\mu_{0}=(19.6 \pm 0.4) \mathrm{cm}^{2} / \mathrm{V}-\mathrm{sec}$. This agreement with previous measurements supports the validity of the reversed field technique in eliminating ion injection effects.

The ion mobilities may be calculated from the "pure polarization" theory which assumes that the only interaction between ion and atom is the attractive force between a point charge and the dipole induced in the target atom. For this case the reduced mobility is independent of the chemical 
nature of the ion and is given by $(6)$

$$
\mu_{0}=0.51\left(1+m_{a} / m_{i}\right)^{\frac{1}{2}} /(N(\epsilon-1))^{\frac{1}{2}},
$$

where $\mathrm{N}$ is the gas density, the dielectric constant of the gas, and $\mathrm{m}_{2}$ and $m_{i}$ the masses of gas atom and ion, respectively. For the polarization attraction case the ionatom collision frequency is independent of energy, with the result that $\mu_{0}$ should be independent of temperature and of $E / p$. The fact that the mobility data of Fig. 2 show little dependence on $\mathrm{E} / \mathrm{p}$ should not be regarded as strong support for the simple polarization attraction theory, since the center of mass energy between ion and atom remains nearly thermal in both cases. For $\mathrm{Hg}^{+}$ions at $\mathrm{E} / \mathrm{p}=5$ the ion energy in the laboratory frame is $\sim 0.6 \mathrm{eV}$ according to Wannier's formula $(7)$; however the $c_{\circ} \mathrm{m}$. energy is only $\sim 0.051 \mathrm{eV}$ (thermal energy at $300^{\circ} \mathrm{K}$ is $0.039 \mathrm{eV}$ )。 Similarly, for $\mathrm{U}^{+}$ions at $\mathrm{E} / \mathrm{p}=15$, the lab frame energy is $\sim 3 \mathrm{eV}$, but the c.m. energy is only $0.089 \mathrm{eV}$. (In the present case, the com. energy may be approximated by

$$
\varepsilon_{\mathrm{cm}} \approx \frac{1}{2} \mathrm{~m}_{r}\left(\left\langle v_{i}^{2}\right\rangle+\left\langle v_{a}^{2}\right\rangle\right)
$$

where $m_{r}$ is the ion-atom reduced mass, $\left\langle v_{i}^{2}\right\rangle$ and $\left\langle v_{a}{ }^{2}\right\rangle$ are the averages of the squares of the ion and atom velocities, respectively, and the ion velocity may be replaced by $v_{d}$, the ion drift velocity.)

Using published values of the dielectric constant of helium(8), eq. (2) yields reduced mobilities of $15.4 \mathrm{~cm}^{2} / \mathrm{V}-\mathrm{sec}$ for both $\mathrm{Hg}^{+}$and $\mathrm{U}^{+}$ions in helium. The agreement of this value with our measured thermal energy value for $\mathrm{U}^{+}, \mu_{0}=(16.0 \pm 0.5) \mathrm{cm}^{2} / \mathrm{V}=\mathrm{sec}_{\text {, }}$ is surprisingly good; however, the measured value for $\mathrm{Hg}^{+}, \mu_{0}=(19.4 \pm 0.5) \mathrm{cm}^{2} / \mathrm{Vasec}$ is $\sim 25 \%$ higher. Our results may be compared with the measured mobilities of alkali ions in helium (9) to see if there is any trend with ion mass for simple ions. 
According to the pure polarization theory, eq. (2), if one compares the mobility of different ions moving in the same gas, the quantity

$$
\mu_{0}^{\prime} \equiv \mu_{0} \mathrm{~m}_{\mathrm{r}}^{\frac{1}{2}}
$$

should be invariant (6). For the case of helium the predicted value from eqs. (2) and (4) is $\mu_{0}^{\prime}=31 \mathrm{~cm}^{2} \mathrm{~V}^{\mathrm{m}} \mathrm{sec}^{-1} a m u^{\frac{1}{2}}$, in good agreement with the measured value for uranium ions $\mu_{0}{ }^{\prime}=(32 \pm 1)$. However, as will be seen from Fig. 3, the data for the alkali ions $\mathrm{Li}^{+}$through $\mathrm{Cs}^{+}$lie substantially above the theoretical value indicated by the dashed line, as does the value for $\mathrm{Hg}^{+}$.

Larger observed mobilities than are predicted by the polarization attraction theory are usually explained in terms of the short-range repulsive interaction between fon and atom, which can not be neglected in a weakly polarizable gas such as helium. (In contrast, agreement between measured and calculated $\mu_{0}{ }^{\prime}$ values and near-invariance of $\mu_{0}^{\prime}$ with alkali ion mass have been noted in the more polarizable noble gases argon, krypton and xenon $(9)_{0}$ ) The onset of the repulsive interaction at moderate ion-atom separations $R$ weakens the $\mathrm{R}^{-4}$ polarization attractive potential, with the result that the scattering angles are somewhat reduced and the mobilities are increased above the pure polarization values. Moreover, inclusion of the repulsive potential in the theory leads to prediction of observable temperature dependences for the mobilities $(6,10)$, a point which should be tested in future experiments. Inasmuch as accurate calculations of the repulsive potentials between ion and atom are not generally available, it is difficult to assess the reason for the discrepancy between theory and experiment for the case of $\mathrm{Hg}^{+}$in helium. It appears, however, that the mobility of $U^{+}$ions in helium is adequately described by the simple "small ion" polarization theory represented 
by eq. (2), even though $\mathrm{U}^{+}$is one of the larger ions studied. Thus, the observation of substantially different mobilities in helium for two heavy ions of comparable mass, $\mathrm{Hg}^{+}$and $\mathrm{U}^{+}$, provides a test for theoretical calculations on comparatively simple ion-atom systems. 


\section{References}

1. R. Johnsen and M. A. Biondi, J. Chem。 Phys., in press.

2. M. A. Biondi, Phy.s. Rev. 20, 730 (1953).

3. I. M. Chanin and M. A. Biondi, Phys。 Rev. 107, 1219 (1957)。

4. M. A. Biondi and I. M. Chanin, Phys. Rev。 94, 910 (1954)。

5. D. L. Albritton, T. M. Miller, D. Wo Martin, and E. W. McDaniel, Phys. Rev. 171, 94 (1962)。

6. P. Langevin, Ann. Chim。 et Phys. 5, 245 (1905).

7. G. H. Wannier, Bell System Tech. J. 32, 170 (1953)。

8. Landolt-Börnstein, Atom und Molekularphysik, 1 Teil, Atome und Ionen, edited by A. Eucken (Springer-Verlag, Berlin, 1950)。

9. A. M. Tyndall, The Mobility of Positive Ions in Gases (Cambridge University Press, Iondon, 1938)。

10. H. R. Hassé and W. R. Cook, Phil. Mag。12, 554 (1931)。 


\section{Figure Captions}

Fig, 1 Simplified diagram of the drift mobility tube/mass spectrometer apparatus.

Fig. 2 Reduced mobilities (at a standard gas density of $2.69 \times 10^{19} \mathrm{~cm}^{-3}$ ) of mass-identified ions in helium. Mercury data taken at pressures of 1.2 Torr (a) and 1.85 Torr $(x)$ and at $T=292^{\circ} \mathrm{K}$. Uranium data taken at 2.0 Torr $(\Delta), 0.96 \operatorname{Torr}(+)$, and $0.98 \operatorname{Torr}(0)$ at $\mathrm{T}=305^{\circ} \mathrm{K}$ 。

Fig. 3 Comparison of the measured mobilities of various ions by means of the quantity $\mu_{0}{ }^{\prime} \equiv \mu_{0} m_{r}^{\frac{1}{2}}$. The theoretical value $\mu_{o}{ }^{\prime}=3 I$ is represented by the dashed line (see text for details). 


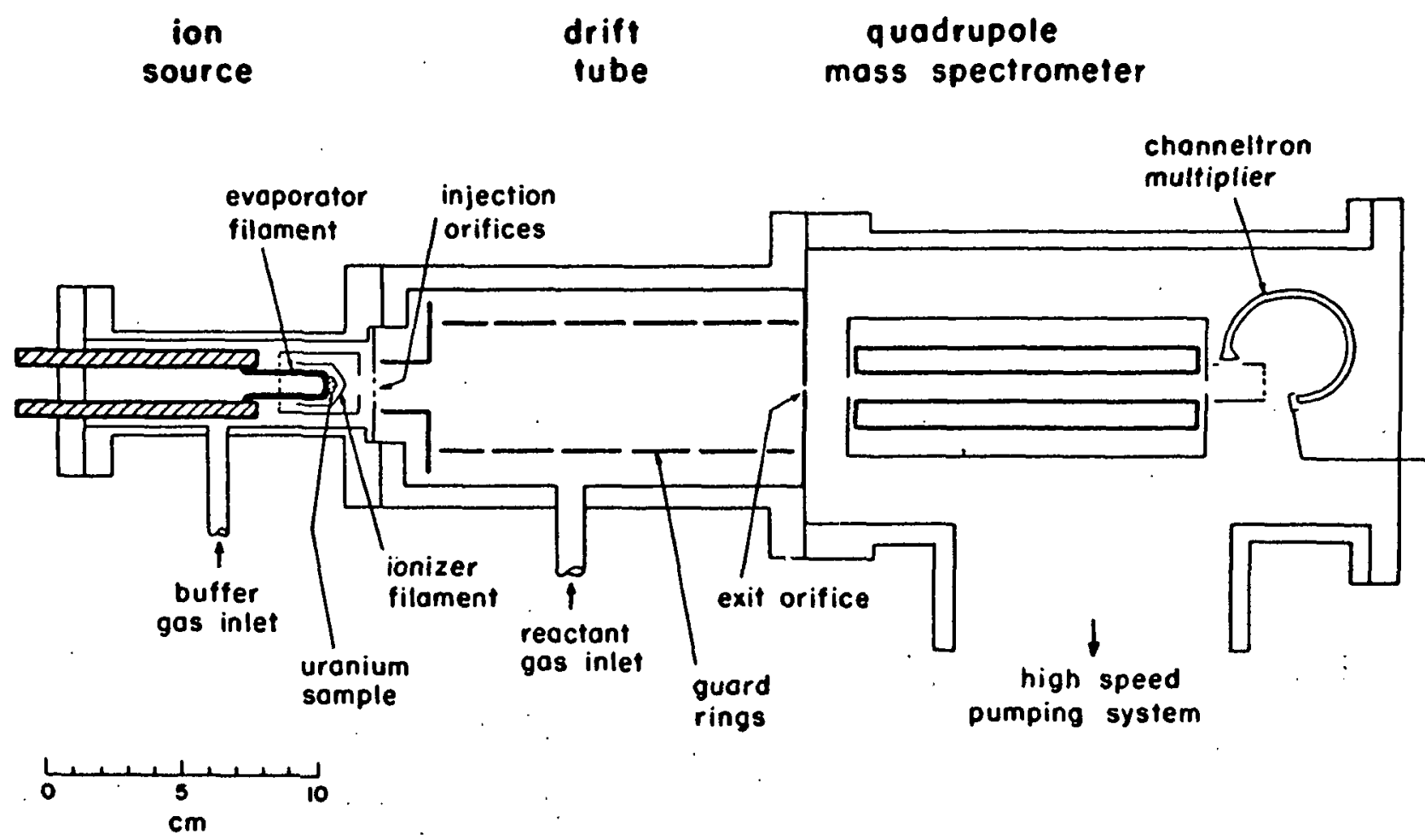

Fig. I Simplifled diagram of the drift woblity tube/mass spectrometer apparatus. 


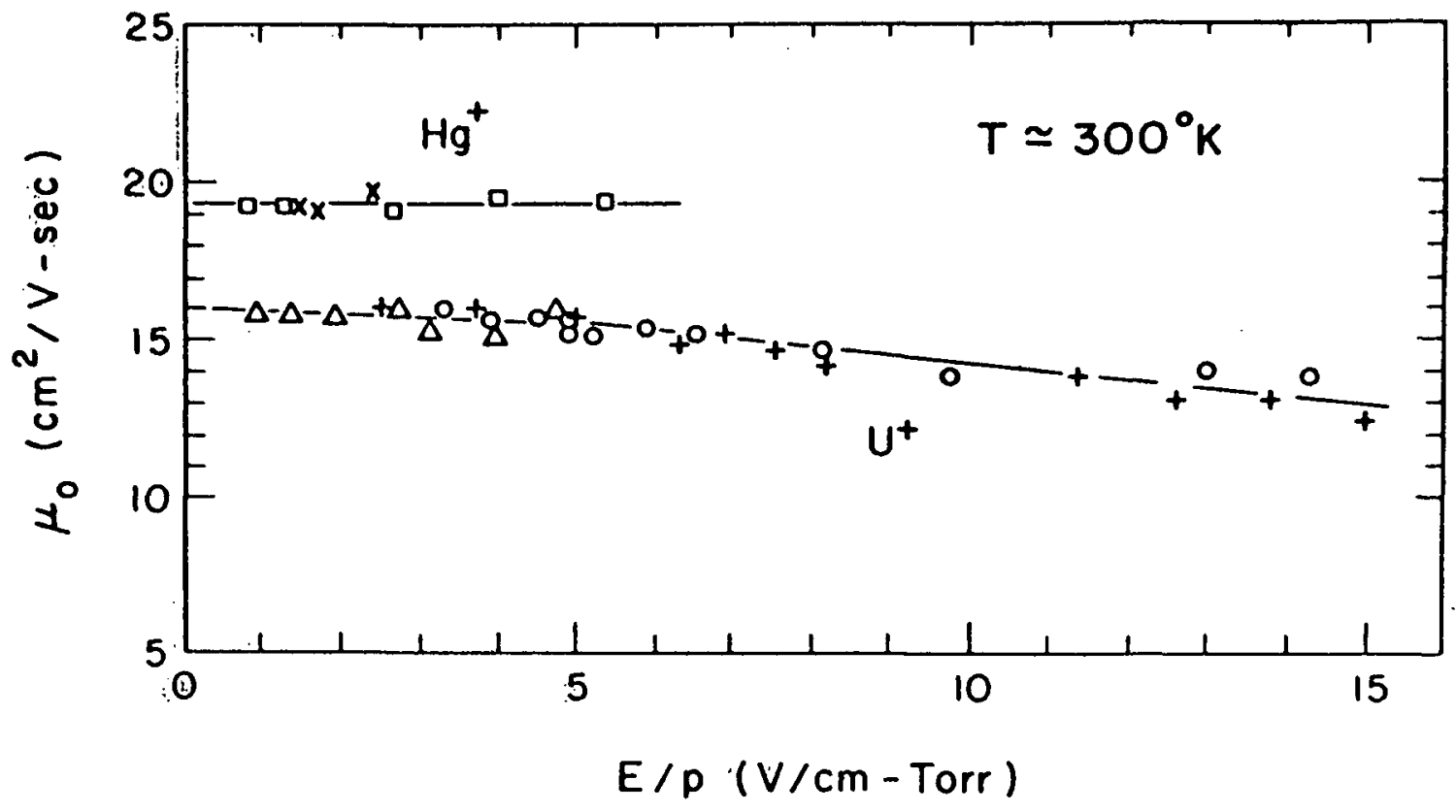

Fig. 2 Reduced mobilities (at a standard gas density of $2.69 \times$ $1019 \mathrm{~cm}^{-3}$ ) of mass-identified ions in helium. Mercury data taken at pressures of 1.2 Torr ( $\bullet$ ) and 1.85 Torr (x) and at $\mathrm{T}=292^{\circ} \mathrm{K}$ 。 Uraniura date taken at 2.0 Torr $(\Delta)$, 0.96 Torr $(t)$, and 0.98 Torr $(0)$ at $\mathrm{T}=305^{\circ} \mathrm{K}$. 


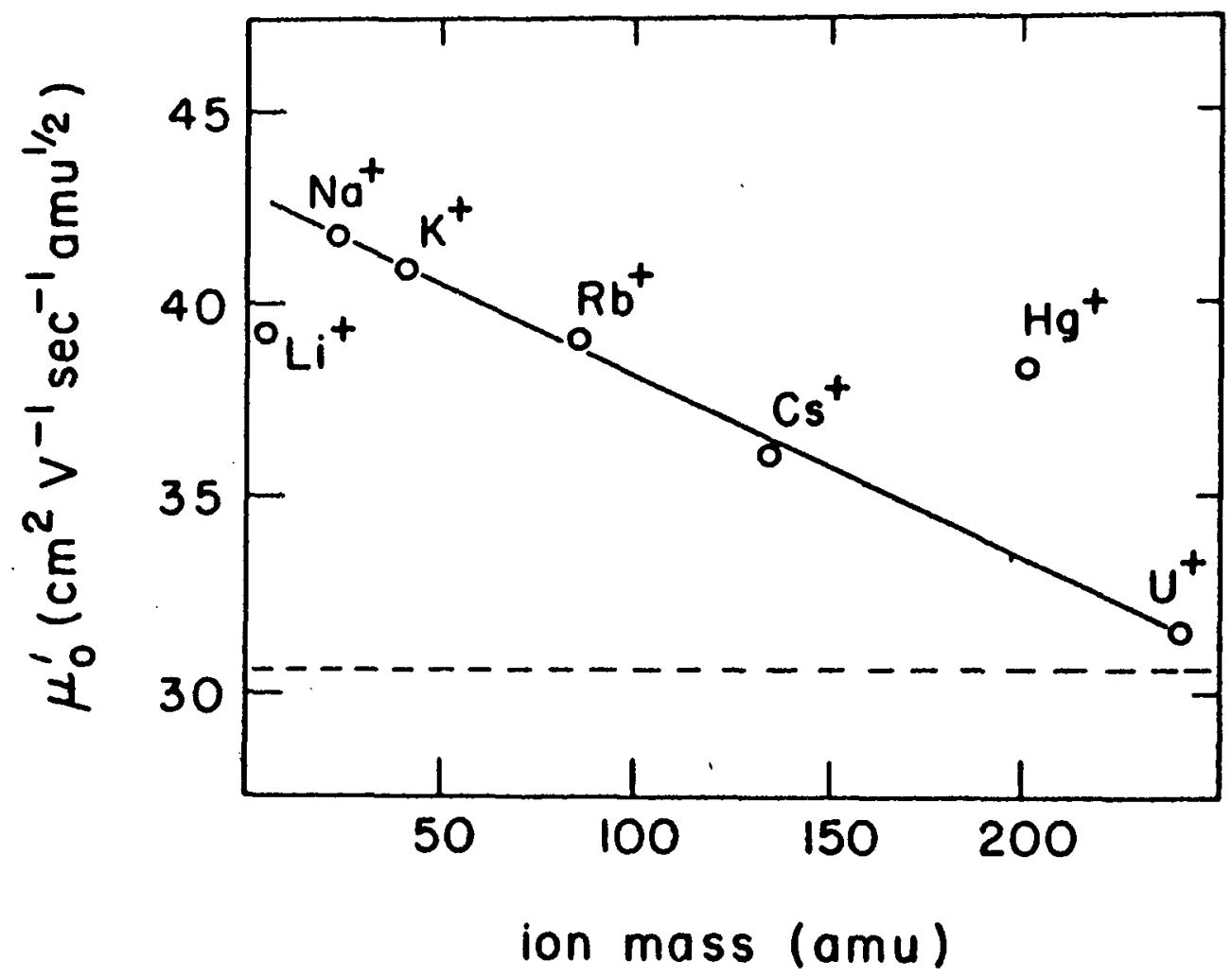

Fig. 3 Comparison of the measured moblilties of various ions by means, of the quantity $\mu_{0}^{\prime}=\mu_{0}{ }^{\frac{1}{2}}$. The theoretical value $\mu_{0}^{\prime}=31$ is represented by the dashed line (see text for detalis). 


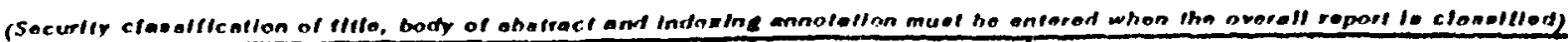
T ODIGINATING ACTIVITY (Comporalo aribios)

University of Pittsburgh

Pittsburgh, Pa. 15213

3 REPOAT TITE

Mobilities of Uranium and Mercury Ions in Helium

4. DESCRIPTIVE NOTES (Type ol report and Inc/uolue dateos)

Scientific paper - issued August 23, 1972

- AU THOAISI (Firal namo, olddlo Inillal, leol naono)

Rainer n.m.i. Johnsen and Manfred A. Biondi

\begin{tabular}{|c|c|c|}
\hline $\begin{array}{l}\text { AEPOAT DATE } \\
\text { August } 1972\end{array}$ & $\begin{array}{c}\text { 7a. TOTAL NO. OF PAGES } \\
13\end{array}$ & $\begin{array}{c}\text { 7b. No. OF hers } \\
10\end{array}$ \\
\hline $\begin{array}{l}\text { B. CONTAACT OA GRANT NO } \\
\text { DA-31-124-ARO-D-440 } \\
\text { b. PROJECr No } \\
\text { P-6563-P }\end{array}$ & $\begin{array}{l}\text { W. OAIOINATOA'S MEPOAT } \\
\text { APP-35 }\end{array}$ & tents) \\
\hline ๙. ARPA Order 826, Am. 9 & 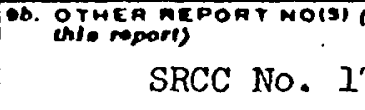 & 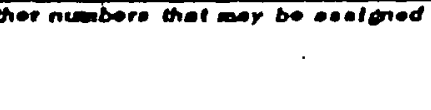 \\
\hline
\end{tabular}

10 DISTAIBUTION STATEMENT

Approved for public release; distribution unlimited.

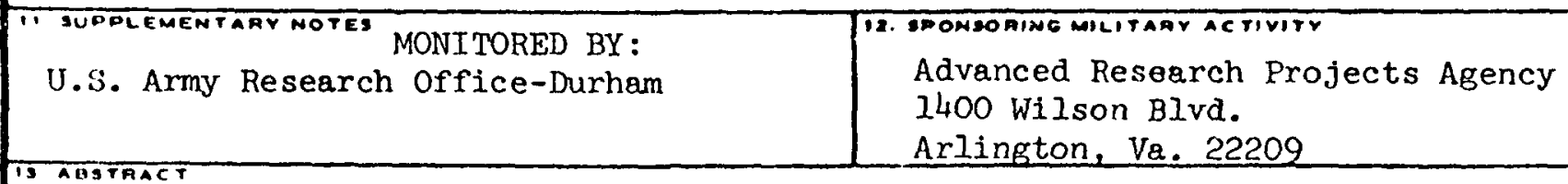

The mobilities of mass-identified $\mathrm{U}^{+}$and $\mathrm{Hg}^{+}$ions in helium have been determined in a drift tube-mass spectrometer apparatus. For uranium ions a reduced mobility value $\mu_{0}=(16.0 \pm 0.5) \mathrm{cm}^{2} / \mathrm{V}-\mathrm{sec}$ is obtained at $305^{\circ} \mathrm{K}$ and a standard gas density of $2.6 \mathrm{C}$ $x 1019 \mathrm{~cm}-3$. The mobility of mercury ions is $\mu_{0}=(19.4 \pm 0.5) \mathrm{cm}^{2} / \mathrm{V}-\mathrm{sec}$ at $292^{\circ} \mathrm{K}$, in agreement with two previous determinations. The effect of fast ion injection in drift mobility measurements is discussed, and a new technique to circumvent these protlems is described. The results are compared with existing theories of ion mobilities. 
UNCTASSTFTED

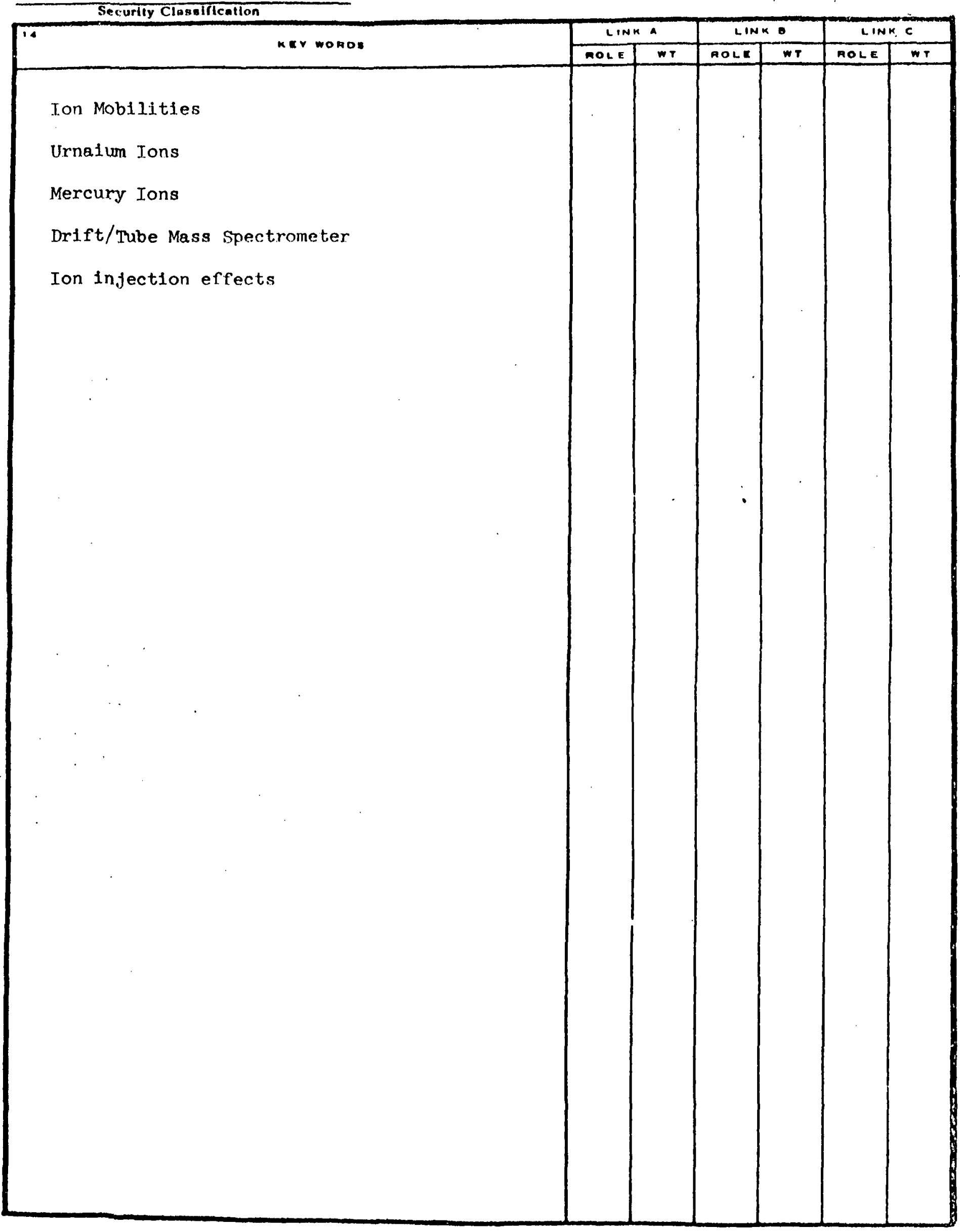

UNCIASSTETED

Securtty Clasollication 